\title{
Microlensing towards the Magellanic Clouds: Nature of the Lenses and Implications for Dark Matter
}

\author{
Kailash C. Sahu \\ Space Telescope Science Institute, Baltimore, MD 21218
}

\begin{abstract}
A close scrutiny of the microlensing results towards the Magellanic clouds reveals that the stars within the Magellanic clouds are major contributors as lenses, and the contribution of MACHOs to dark matter is 0 to $5 \%$. The principal results which lead to this conclusion are the following.

(i) Out of the $\sim 17$ events detected so far towards the Magellanic Clouds, the lens location has been securely determined for one binary-lens event through its caustic-crossing timescale. In this case, the lens was found to be within the Magellanic Clouds. Although less certain, lens locations have been determined for 3 other events and in each of these 3 events, the lens is most likely within the Magellanic clouds.

(ii) If most of the lenses are MACHOs in the Galactic halo, the timescales would imply that the MACHOs in the direction of the LMC have masses of the order of $0.5 \mathrm{M}_{\odot}$, and the MACHOs in the direction of the SMC have masses of the order of 2 to $3 \mathrm{M}_{\odot}$. This is inconsistent with even the most flattened model of the Galaxy. If, on the other hand, they are caused by stars within the Magellanic Clouds, the masses of the stars are of the order of $0.2 \mathrm{M}_{\odot}$ for both the LMC as well as the SMC.

(iii) If $50 \%$ of the lenses are in binary systems similar to the stars in the solar neighborhood, $\sim 10 \%$ of the events are expected to show binary characteristics. The fact that the two observed binary events are caused by lenses within the Magellanic Clouds would imply that there should be a total of $\sim 20$ events caused by lenses within the Magellanic Clouds. This implies that most of the microlensing events observed so far are probably caused by stars within the Magellanic Clouds. (iv) If the microlensing events are caused by MACHOs of $0.5 \mathrm{M}_{\odot}$, as claimed from the LMC observations, about 15 events should have been detected by now towards the SMC, with timescales of $\sim 40$ days. The fact that no event has been detected towards the SMC caused by MACHOs (both the events detected towards the SMC have been shown to be due to self-lensing) places severe constraints on the MACHO contribution and suggests that the contribution of MACHOs to dark matter is consistent with zero, with an upper limit of $5 \%$.
\end{abstract}




\section{Introduction}

Most spiral galaxies are known to have flat rotation curves (e.g. Begeman et al. 1991). In the outer parts of the galaxies in particular, the visible matter falls far short of what is required to explain the flat rotation curves. In our own Galaxy, the rotation curve is observed to be flat up to at least $16 \mathrm{kpc}$ from the center (e.g. Fich et al. 1989). The scenarios proposed to explain the flat rotation curves are either departure from Newtonian dynamics in large scale, or presence of dark matter (for a review see Sanders 1990). In the latter and more conventional scenario, the rotation curves would imply that a significant part of the mass of the galaxies, including our own, resides in the halo in some form of dark matter. The nature of this dark matter has been hypothesized to be in either of the two forms: MACHOs (i.e. massive compact halo objects which is a collective term for 'Jupiters', brown dwarfs, red dwarfs, white dwarfs, neutron stars or black holes); or elementary particles such as massive neutrinos, axions or WIMPs (weakly interacting massive particles such as photinos etc.). One important distinguishing factor of the MACHOs is that they can have detectable gravitational effects and can cause "gravitational microlensing" of background stars.

Einstein (1936) was the first to point out that a star can act as a gravitational lens for another background star, if the two are sufficiently close to each other in the line of sight. Given the observational capabilities of that time, Einstein had however considered this to be a purely theoretical exercise since there was "no hope of observing such a phenomenon directly".

Paczyński (1986) worked out the probability of such microlensing events by MACHOs and showed that if the halo of our Galaxy is made up of MACHOs, the probability of finding them is $5 \times 10^{-7}$, independent of their mass distribution. He suggested an experiment to look for such events using the LMC stars. Such an experiment was taken up by 2 groups who reported their first results in 1993 (Alcock et al. 1993; Aubourg et al. 1993). In 6 years' monitoring of millions of stars towards the Magellanic Clouds, so far about 17 events have been detected towards the Large Magellanic Cloud (LMC) and 2 events towards the Small Magellanic Cloud (SMC).

The observed microlensing optical depth as derived from these observations is $\sim 1 \times 10^{-7}$. This is about a factor of 5 lower than what would be expected if the dark matter is entirely made up of MACHOs. To complicate the issue further, the simple microlensing light curves cannot tell us the location of the lenses because of a degeneracy between the distance, mass and the proper motion of the lens. So the lenses can be stars in the Galactic disk (Gould 1994, Evans et al. 2001, Gates and Gyuk, 2001), MACHOs in the Galactic halo (Alcock et al. 2000a), or stars within the LMC itself (Sahu 1994a,b; Evans and Eamonn 2000). As 
a result, the interpretation of these lensing events, and their locations in particular, have remained uncertain.

\section{Overview of the Problem}

The contribution of known stars to the microlensing optical depth had not been calculated until after the first microlensing events were reported towards the LMC. Such calculations revealed that the microlensing optical depth due to the known stars within the LMC is close to the observed optical depth, particularly in the region of the bar where the microlensing event was detected (Sahu 1994a,b; Wu 1994). This was used to argue that the stars within the Magellanic Clouds must play a significant role as lenses.

Lensing by stars within the Magellanic clouds is now commonly referred to as "selflensing". It is known for a long time (e.g. Schneider et al. 1992), and it was re-derived by Gould (1995) that the self-lensing microlensing optical depth can be expressed as a function of the velocity dispersion

$$
\tau=2 \times \sec ^{2}(\theta) v^{2} / c^{2}
$$

where $\theta$ is the inclination of the disk of the LMC (the best estimated value of which is $34.7 \pm 6 \mathrm{deg}$, van der Marel and Cioni, 2001). Since the velocity dispersion of the stars in the disk of the LMC is about $20 \mathrm{~km} / \mathrm{s}$, the above equation would imply that the self-lensing optical depth is about $2 \times 10^{-8}$, which is too low to explain the observed microlensing events.

However, there are some problems in such an approach. First, eq. (1) can be applied only for a virialized system. The Magellanic Clouds are not relaxed, and far from virialized systems: they are dynamically disturbed, they show tidal-tail structures, and the velocity dispersions are different for low and high-mass objects (for a review, see Westerlund 1997). Second, the lenses are low-mass objects for which the velocity dispersions are unknown. Third, the velocity dispersions in the region of the bar, where most of the events have been found so far, is also unknown.

A cautionary note seems appropriate here. A similar story had repeated towards the Galactic bulge. The velocity dispersion of the Galactic bulge stars would imply a microlensing optical depth which is a factor of at least 3 smaller than the observed optical depth. In the case of the Galactic bulge, the re-discovery of the Galactic bar, with an inclination of $15^{\circ}$ to

the line of sight helped in solving the puzzle of the discrepancy between the observed and the expected microlensing optical depth (Kiraga and Paczyński, 1994; Paczyński et al. 1995). 
In the meantime, several papers have appeared with detailed calculations of the optical depth taking into account the extra effects such as 'diffusion' of small-mass objects, which causes the velocity dispersion of less massive objects to be larger (e.g. Salati et al. 1999).

The full range of the calculated optical depth due to the stars within the LMC, as published in the literature so far, is $1 \times 10^{-8}$ to $2 \times 10^{-7}$ (e.g. Gyuk et al. 2000, Graff 2001, Salati et al. 1999, Aubourg et al. 1999). The high end of this self-lensing optical depth would imply that all the observed events are caused by stars within the LMC and that the contribution of MACHOs to the dark matter is negligible. The low-end of the self-lensing optical depth would imply that none of the observed events are caused by stars within the Magellanic clouds, and hence the events are caused most likely by MACHOs in the halo. In the extreme case where all the events are caused by MACHOs in the Galactic halo, the implied contribution of MACHOs to dark matter is about 25\% (Alcock et al. 2000a, Afonso et al. 2002).

Cleary, it is important to know the exact location and nature of the lenses, which can shed some light on the whether MACHOs can account for the long-sought dark matter in the halo. We obviously need some observational tests to guide us in resolving this puzzle. The rest of the paper mainly deals with such tests and the current status of the results from these tests.

It is worth pointing out here that, in the self-lensing hypothesis, it is implicit that the sources and the lenses reside within the LMC. The sources cannot be considered as background sources since the derivation of the observed microlensing optical depth assumes that all the monitored stars which are within the Magellanic clouds contribute to the microlensing optical depth.

\section{Observational Tests}

The simple microlensing light curve is inadequate to the determine the lens location because of a degeneracy between the distance, the proper motion and the mass of the lens. A typical difficulty is that, in order to determine the lens location, both the mass and the proper motion of the lens are needed. Generally, these quantities can only be estimated statistically, since the lens is not detected directly. As a result, the interpretations of these lensing events, and the lens locations in particular, remain uncertain.

Fortunately, there are several observational tests which can be used to infer the nature of the lenses, including some tests which can be used for direct determinations of their locations. The observational tests that I will discuss here, some of which have already provided 
clear results, are the following:

(i) Determination of lens location through "caustic-crossing" timescale

(ii) Other direct determinations of lens locations

(iii) Frequency of observed binary-lens events

(iv) Timescales of SMC vs. LMC events

(v) Spatial distribution of the microlensing events

\section{1. "Caustic-crossing" timescale}

Clearly, the best test would be to measure the location of the lens directly. In a few special cases, such a direct determination of the lens location is indeed possible. One such special case is when the lens is a binary, and the source crosses the caustics produced by the binary lens. Since a caustic is essentially a straight line in space, the time taken by the caustic to cross the source provides a direct measure of the proper motion of the lens projected onto the source plane. If the lens is in the halo at a distance of $\sim 10 \mathrm{kpc}$, then the expected proper motion of the lens is about $200 \mathrm{~km} / \mathrm{s}$ which, projected onto the source plane, is about $1000 \mathrm{~km} / \mathrm{s}$. So the time for the caustic to cross the source would be of the order of half an hour. If, on the other hand, the lens is within the Magellanic Clouds, the expected proper motion is about $50 \mathrm{~km} / \mathrm{s}$, and hence the caustic crossing time is expected to be of the order of 10 hours. Thus, monitoring a caustic crossing provides a powerful method to determine the location of the lens.

\subsection{1. $M A C H O ~ 98-S M C-1$}

Such an opportunity presented itself after the first binary-lens event, MACHO 98-SMC1, was discovered by the MACHO collaboration in 1998 (Alcock et al. 1999). After the first caustic crossing was reported, the PLANET collaboration, with its 24-hour access to telescopes around the world, predicted a second caustic crossing and began monitoring this event, with a particular interest in fully sampling the second caustic crossing (Albrow et al. 1999). The time for the caustic to cross the source was found to be 8.5 hours (Fig. 1), which demonstrated that the lens is within the SMC (Fig. 2). The result was further confirmed by the EROS, MACHO and MPS collaborations, who also concluded that the lens is most likely within the SMC (Afonso et al. 1998, 2000, Alcock et al. 1999, Rhie et al. 1999). 


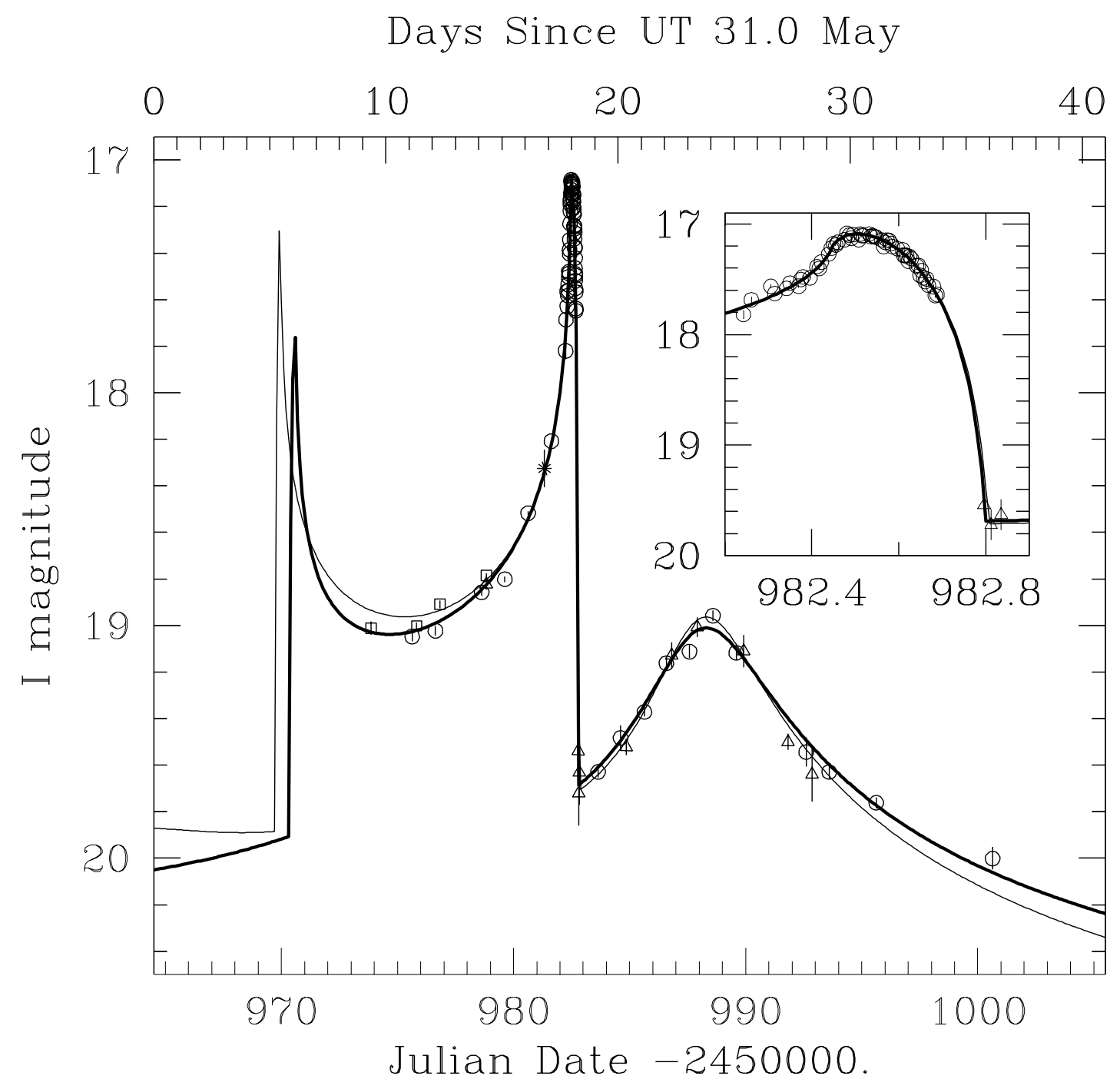

Fig. 1. - Light curve of the PLANET data for MACHO-98-SMC-1. Shown are the data from the SAAO $1 \mathrm{~m}$ (circles), the CTIO $0.9 \mathrm{~m}$ (squares), the CTIO-Yale $1 \mathrm{~m}$ (triangles), and the Canopus $1 \mathrm{~m}$ (asterisks). The inset covers about 0.6 days, corresponding to less than one tick mark on the main figure. (Taken from Albrow et al. 1999) 


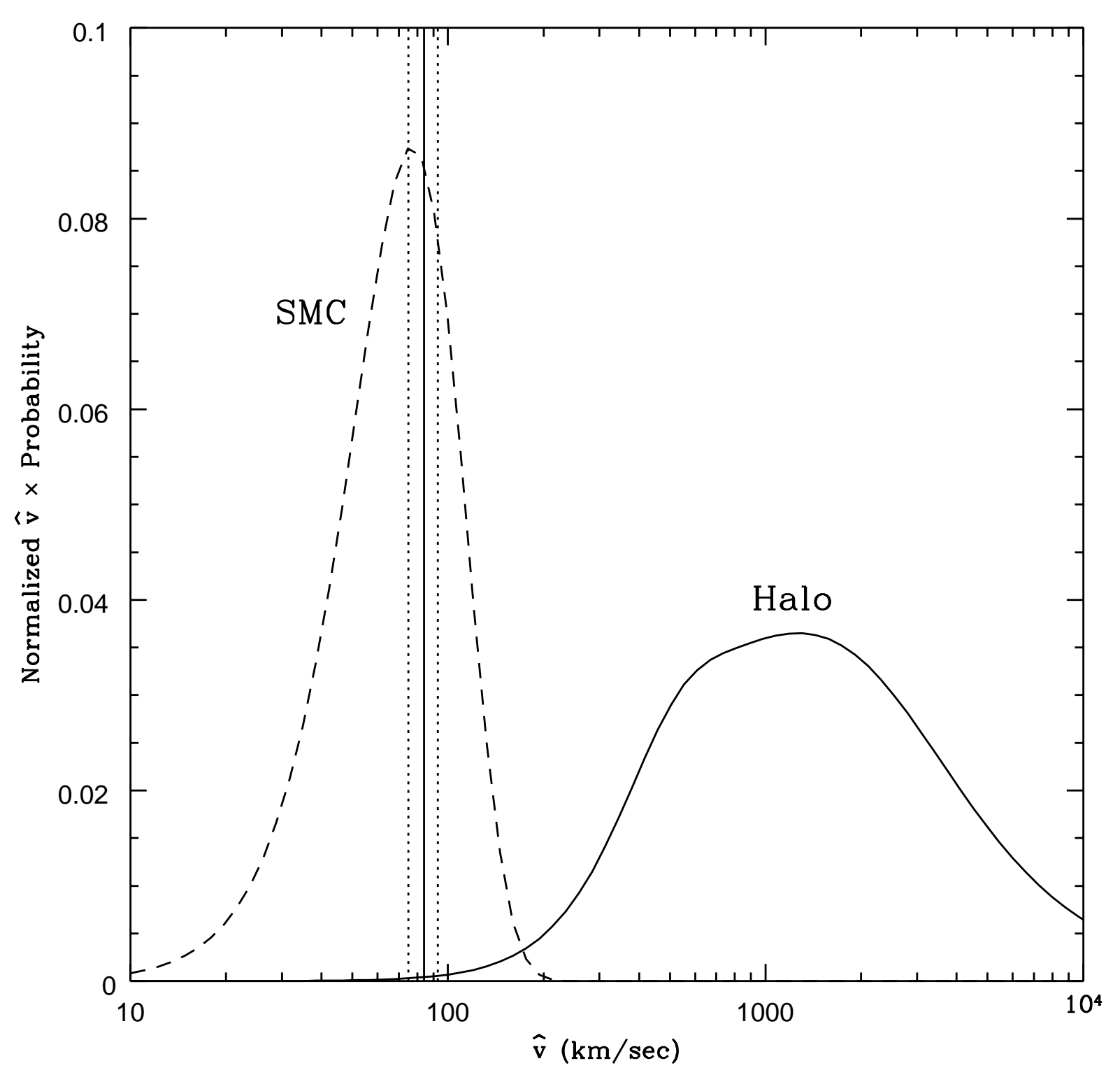

Fig. 2.- Predicted velocity distributions (at the source plane) for halo and SMC lenses. The measured value of the velocity and error bars are shown as vertical lines. The observed value is inconsistent with the halo hypothesis. The lens and source could possess normal SMC kinematics. (Taken from Alcock et al. 1999) 


\subsection{2. $M A C H O-L M C-9$}

The second binary-lens event, MACHO-LMC-9, was discovered by the MACHO collaboration towards the LMC, for which the observations are not as dense (Alcock et al. 1997a). The caustic crossing timescale of the event also suggests that the lens is most likely within the LMC (Fig. 3), although the sparse sampling of this event quality has led to some doubt as to whether this is a microlensing event at all (Alcock et al. 2000a,b).

\subsection{Other determinations of lens locations}

\subsubsection{MACHO 97-SMC-1}

97-SMC-1 is the first microlensing event observed towards the SMC, which has a large timescale of 220 days, in contrast to the average timescale of 40 days as observed for the more than dozen microlensing events towards the LMC (Alcock et al. 1997b).

If the lens is a MACHO in the Galactic halo, the timescale of the event would suggest that the mass of the lens is about $3 \mathrm{M}_{\odot}$. Clearly, this cannot be a normal star of $3 \mathrm{M}_{\odot}$ since it would be too bright and would be easily detectable. Indeed, a spectrum of the source obtained by Sahu and Sahu (1999) shows the spectrum to be a pure B-type spectrum as expected from a source within the SMC, without any contribution from the lens (Fig. 4). This would suggest that the lens is either a black hole in the Galactic halo, or a small mass object within the SMC.

A black hole in the Galactic halo is an unsatisfactory explanation since that would imply that the MACHOs in the direction of the LMC are predominantly 0.5 solar mass objects and the MACHOs in the direction of the SMC are predominantly 3 solar mass objects. The locations of the LMC and the SMC too close to permit such a mass segregation in these two directions even in the most extreme flattened model of the Galaxy. This leaves us with the only other alternative that the lens must be a small mass object within the SMC.

Furthermore, as explained in the next section, this long timescale of the SMC events is precisely what one would expect if the lenses are within the Magellanic Clouds, which further supports the view that the lens is most likely within the SMC. 


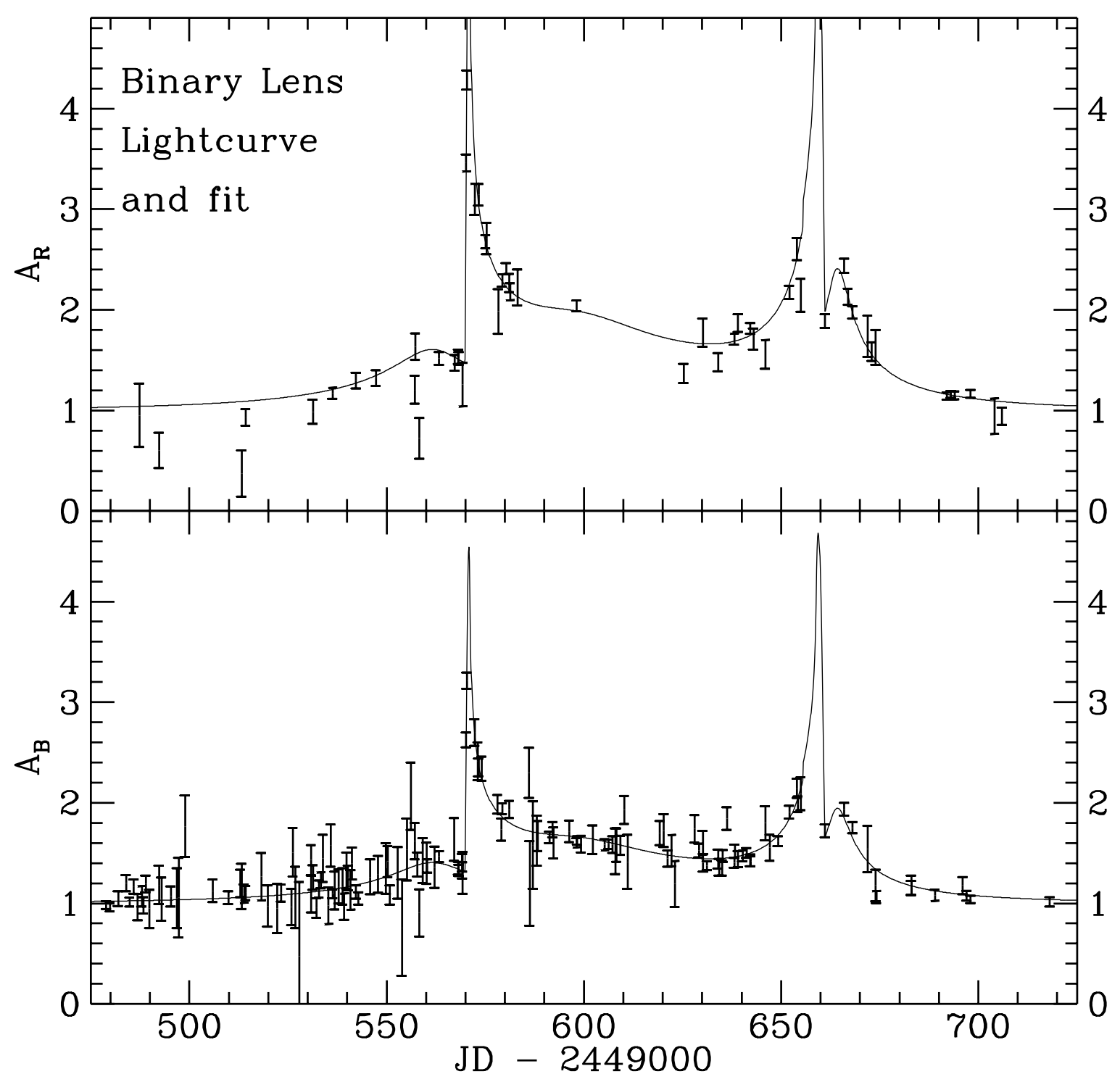

Fig. 3.- Light curve of LMC-9 in the around the peak of the event, and the best-fit binary lens light curve. The most likely lens location is within the LMC. (Taken from Alcock et al. 1997a). 


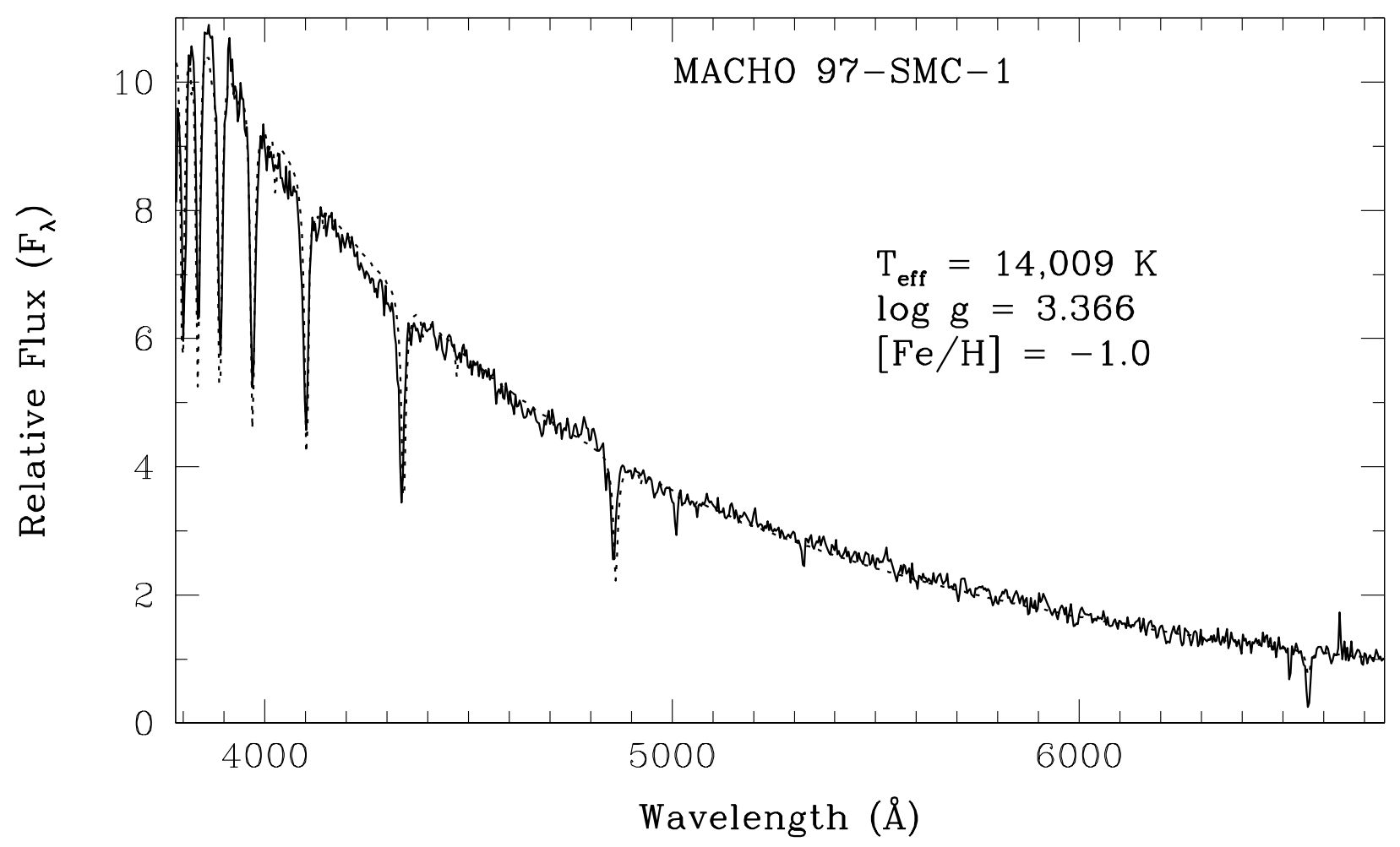

Fig. 4.- Observed spectrum of MACHO 97-SMC-1 taken on 30th May 1997, just after the source had crossed the Einstein ring radius of the lens (solid curve), along with the best fit stellar model spectrum (dashed line). The contribution from the lens is negligible which, combined with the microlensing time scale, implies that the lens must be within the SMC. (Taken from Sahu and Sahu, 1998). 


\subsection{2. $M A C H O ~ 96-L M C-2$}

Lens locations have been estimated for another event MACHO 96-LMC-2, where the source is a binary (Alcock et al. 2001a). In this case too, the lens is most likely within the LMC.

Thus there are 4 cases so far, for which we know the locations of the lenses. In all the cases, without exception, the most likely locations of the lenses are within the Magellanic Clouds.

It is worth noting here that the lens location has been recently determined for an event caused by a star in the local Galactic disk (Alcock et al. 2001b). The events caused by nearby lenses are expected to exhibit "parallax" effects which are easier to detect. The fact that only one event has been detected caused by a star in the local Galactic disk is consistent with the expected frequency of nearby stars acting as lenses.

\subsection{Frequency of observed binary-lens events}

For the sake of argument, let us start with the assumption that all the events except the two binary events for which we know that the lenses are within the Magellanic clouds are caused by MACHOs in the halo. This would be quite extra-ordinary since this would mean that the only two events that are caused by the stars within the Magellanic clouds not only happen to be binaries, but they also show caustic crossing structure in their light curves. If one event caused by a star in the Magellanic clouds is observed, the probability that the lens would be a binary is about 50\% (assuming 50\% of the lenses are in binary systems similar to the stars in the solar neighborhood). Since only $20 \%$ of the binary-lens events are expected to show caustic-crossing structures (which is borne out by the observations towards the Galactic bulge as well), the probability that it would show a caustic-crossing structure is $10 \%$. The probability that a second event caused by a Magellanic cloud star would again be a binary and show caustic structure is again $10 \%$. The probability that two successive events caused by Magellanic cloud stars will both be binaries and show caustic crossing structures is thus $1 \%$, and hence extremely unlikely. We can follow the same argument in reverse and ask the following question: if 2 events which show binary characteristics are within the Magellanic clouds, how many of the observed events should be caused by stars within the Magellanic clouds? Since 10\% of the events are expected to show binary characteristics, one would expect about 20 events to be caused by stars within the Magellanic clouds. This is

more or less the number of events observed, which suggests that most of the lenses must be within the Magellanic clouds. 


\subsection{Timescales of SMC vs. LMC events}

The relative timescales of the LMC and the SMC events provide further indications on the locations of the lenses. If the lenses are MACHOs in the Galactic halo, the characteristics of the lenses towards the LMC and the SMC are expected to be the same and hence both the LMC and the SMC events are expected to have similar timescales. On the other hand, if the lenses are within the Magellanic Clouds, the expected timescales of the LMC and the SMC events are expected to be very different. The LMC has a depth of less than $1 \mathrm{kpc}$ in a typical line of sight whereas the SMC as a much larger depth of about $5 \mathrm{kpc}$ along a typical line of sight. So the typical distance between the lens and the source is much smaller in the case of LMC than in the case of the SMC. As a result, the size of the Einstein ring (which scales as the square root of the distance between the source and the lens) is much smaller for the LMC events than than for the SMC events. Since the velocity dispersions of the stars within the LMC and the SMC are similar, the timescales of the SMC events are expected to be much longer than the LMC events, if the lenses are within the Magellanic Clouds.

Table 1: Lens masses for different scenarios

\begin{tabular}{ccccc}
\hline $\begin{array}{c}\text { Line of } \\
\text { Sight }\end{array}$ & No. of & $\mathrm{M}_{\text {lens }}($ halo $)$ & $\mathrm{M}_{\text {lens }}(\mathrm{LMC} / \mathrm{SMC})$ & $\mathrm{M}_{\text {lens }}$ (local disk) \\
\hline LMC & $\sim 17$ & $\left(M_{\odot}\right)$ & $\left(M_{\odot}\right)$ & $\left(M_{\odot}\right)$ \\
SMC & $\sim 2$ & $\sim 0.5$ & $\sim 0.2$ & $\sim 0.5$ \\
\hline
\end{tabular}


Two events have been observed towards the SMC so far which have timescales of 75 and 125 days. This would correspond to lens masses of 2 to 3 solar masses if the lenses are in the halo, and about 0.2 to 0.3 solar masses if the lenses are within the Magellanic clouds. For the $\sim 17$ events observed towards the LMC, the timescales are much shorter, which would correspond to lens masses of $\sim 0.5$ solar masses if the lenses are in the halo, and $\sim 0.2$ solar masses if the lenses are within the LMC. This is shown it tabular form in Table 1. If the events are predominantly caused by MACHOs in the halo, one faces the unrealistic consequence that the MACHOs in the direction of the LMC and the SMC are of very different mass. Self-lensing is the only scenario which gives consistent masses for both the LMC and the SMC events. The longer durations of the SMC events are a natural consequence of the self-lensing hypothesis.

\subsection{Spatial distribution of the events}

Finally, the spatial distribution of the events provide some extra insight into the nature of the lenses. As first pointed out by Sahu (1994 a,b), the events should be more concentrated towards the bar of the LMC if the lensing is caused by the stars within the LMC. If the events are caused by MACHOs then the events (for a given number of monitored stars) should be uniformly distributed over the whole of the LMC. Unfortunately, all the analyses by the MACHO group so far have been confined to the region around the bar of the LMC. The EROS group, however, have mostly monitored in the region outside the bar, and have not detected any event. From their non-detection of microlensing events, they derive a microlensing optical depth which is much smaller than the optical depth determined by the MACHO group. This is consistent with the fact that stars within the Magellanic clouds play a dominant role as lenses.

Monitoring some regions far from the bar of the LMC to look for microlensing events

would provide a clear test on whether the lensing events are caused by MACHOs or stars within the LMC (e.g. Stubbs, 1999).

\section{Implications on Dark Matter}

The most significant insight into the contribution of dark matter, in my view, comes from a comparative study of the number of observed events towards the LMC and the SMC, and their timescales. So far, 2 microlensing events have been detected towards the SMC with time scales of 75 and 125 days. From these two events, the optical depth towards the SMC 
has been estimated to be $\sim 2 \times 10^{-7}$ which is about the same as the optical depth towards the LMC (Alcock et al. 1999). In terms of optical depth, these two events are equivalent to about 15 events with timescales of $\sim 40$ days (optical depth scales as the square of the timescale). Thus, if the microlensing events are caused by 0.5 solar mass MACHOs as claimed from the LMC observations, about 15 such events should have been detected towards the SMC by now. Furthermore, as discussed earlier, both of the observed SMC events have been shown to be caused by stars within the SMC. Thus no microlensing event caused by a MACHO has been detected towards the SMC, whereas 15 such events should have been detected if the contribution of MACHOs to dark matter is indeed $20 \%$ as claimed from the detection of $\sim 20$ microlensing events towards the LMC. This leads us to the inevitable conclusion that the contribution of MACHOs to dark matter is less than $2 \%$, with a strong upper limit of $5 \%$.

\section{Conclusions}

Results from several observational tests are already available which can be used to clearly distinguish between different scenarios for the nature of the lenses. I have argued that the results obtained so far point to the fact that the observed microlensing events towards the Magellanic clouds are caused by the stars within the Magellanic Clouds. Consequently, the contribution of MACHOs to the dark matter is consistent with zero, with a strong upper limit of $5 \%$. However, some estimates suggest that the known stars within the Magellanic Clouds fall short by a factor of a few to explain the observed microlensing events. The contribution to the microlensing optical depth by the known stars is, however, very uncertain, and further work would be needed to resolve this issue.

References: skip

Afonso, C., et al. 1998, A\& A, 337, L17

Afonso, C., et al. 2000, ApJ, 532, 340

Afonso, C., et al. (EROS Collaboration) 2002, A\& A, in press (astro-ph/0212176)

Albrow, M. et al. 1999, ApJ, 512, 672

Alcock, C. et al. 1993, Nature, 365, 621

Alcock, C. et al. 1997a, ApJ, 491, L11

Alcock, C. et al. 1997b, ApJ, 486, 697

Alcock, C. et al. 1999, ApJ, 518, 44

Alcock, C. et al. 2000a, ApJ, 542, 281

Alcock, C. et al. 2000b, ApJ, 541, 270

Alcock, C. et al. 2001a, ApJ, 552, 259

Alcock, C. et al. 2001b, Nature, 414, 617 
Aubourg, E. et al. 1993, Nature, 365, 623

Aubourg, E. et al. 1999, A\& A, 347, 850

Begeman, K.G., Broeils, A.H., Sanders, R.H., 1991, MNRAS, 249, 523

Einstein, A., 1936, Science, 84, 506

Evans, N. W., Gyuk, G., Turner, M. S., Binney, J., 1998, ApJ, 501, L45

Evans, N. W., Eamonn, K., 2000, ApJ, 529, 917

Fich, M, Blitz, L, Stark, A., 1989, ApJ, 342, 272

Gates, E.L., Gyuk, G., 2001, ApJ, 547, 786

Gould, A. 1995, ApJ, 441, 77

Gould, A., 1994, ApJLett, 421, L71

Graff, D., 2001, Microlensing 2000: A New Era of Microlensing Astrophysics, ASP Conference Proceedings, Vol. 239. Edited by J. W. Menzies and Penny D. Sackett, p.73

Gyuk, G., Dalal, N., Griest, K., 2000, ApJ, 535, 90

Kiraga, M., Paczynski, B., 1994, ApJ, 430, L101

van der Marel, R., Cioni, M.L., 2001, AJ, 122, 1807

Paczyński, B, 1986, ApJ, 304, 1

Paczynski, B., Stanek, K. Z., Udalski, A., Szymanski, M., Kaluzny, J., Kubiak, M., Mateo, M., Krzeminski, W., 1994, ApJ, 435, L113

Palanque-Delabrouille, N., et al. (EROS collab) 1997, Astron. Astrophys. 332, 1

Palanque-Delabrouille, N., 2001, New Astron. Rev., 45, 395

Rhie, S.H. et al. 1999, apJ, 522. 1037

Sahu, K.C. 1994a, Nature, 370, 275

Sahu, K.C. 1994b, PASP, 106, 942

Sahu K.C., Sahu, M.S., 1998, ApJ, 508, 147

Salati, P. et al. 1999, A\& A, 350, L57

Sanders, R.H., 1990, Astron. Astrophys. Rev., 2, 1

Schneider, P, Ehlers, J. and Falco, E.E., 1992, "Gravitational Lensing", published by SpringerVerlag.

Stubbs, C, In The Third Stromlo Symposium: The Galactic Halo, eds. Gibson, B.K., Axelrod, T.S. \& Putman, M.E., ASP Conference Series Vol. 165, p503

Westerlund, B.E., 1997, "The Magellanic Clouds", Cambridge Univ. Press

Wu, X-P. 1994, ApJ., 435, 66 